\title{
STRUCTURAL INFORMATION DETECTION BASED FILTER FOR GF-3 SAR IMAGES
}

\author{
Zengguo Sun ${ }^{1, *}$, Yunjing Song ${ }^{2}$
}

${ }^{1}$ School of Computer Science, Shaanxi Normal University, Xi’an China, duffer2000@163.com

${ }^{2}$ School of Computer Science, Shaanxi Normal University, Xi'an China, 1469279168@qq.com

KEY WORDS: GF-3 SAR images, despeckling filter, adaptive windowing, structural information

\begin{abstract}
:
GF-3 satellite with high resolution, large swath, multi-imaging mode, long service life and other characteristics, can achieve allweather and all day monitoring for global land and ocean. It has become the highest resolution satellite system in the world with the C-band multi-polarized synthetic aperture radar (SAR) satellite. However, due to the coherent imaging system, speckle appears in GF-3 SAR images, and it hinders the understanding and interpretation of images seriously. Therefore, the processing of SAR images has big challenges owing to the appearance of speckle. The high-resolution SAR images produced by the GF-3 satellite are rich in information and have obvious feature structures such as points, edges, lines and so on. The traditional filters such as Lee filter and Gamma MAP filter are not appropriate for the GF-3 SAR images since they ignore the structural information of images. In this paper, the structural information detection based filter is constructed, successively including the point target detection in the smallest window, the adaptive windowing method based on regional characteristics, and the most homogeneous sub-window selection. The despeckling experiments on GF-3 SAR images demonstrate that compared with the traditional filters, the proposed structural information detection based filter can well preserve the points, edges and lines as well as smooth the speckle more sufficiently.
\end{abstract}

\section{INTRODUCTION}

Synthetic aperture radar (SAR) achieve high resolution microwave imaging by the synthetic aperture principle. As an active sensor, the synthetic aperture radar has widely applied in many aspects of society. GF-3 is the first Chinese civil c-band SAR launched on 10 August 2016 by China Academy of Space Technology. And it operates in 12 imaging modes with a fine spatial resolution up to $1 \mathrm{~m}$. However, due to the coherent imaging system, speckle appears in GF-3 SAR images, and it hinders the understanding and interpretation of images seriously. Therefore, the research of SAR image speckle suppression is an important part of the SAR image processing [1,2]. GF-3 SAR images have very useful information such as strong edge, structure information, and point targets. However, the conventional local statistical special filters for SAR images cannot preserve information. The adaptive algorithm used in SAR image reduction is based on local statistical properties. Such as Lee filtering that a linear reduction method is based on the minimum mean square error (MMSE) criterion [3,4] and Gamma MAP filter which is based on maximum a posterior probability (MAP) criterion [5,6]. However, these two methods do not distinguish the different types of the current region. So they cannot effectively suppress the speckle in homogeneous region and edge region and cannot effectively preserve the strong edge, structure information, and the point targets.

To solve the problem, in this paper, the structural information detection based filter is constructed, successively including the point target detection in the smallest window, the adaptive windowing method based on regional characteristics [7], and the most homogeneous sub-window selection $[8,9]$.

\section{DATA ANALYSIS OF GF-3 SAR IMAGES}

GF-3 satellite is a SAR satellite which has the 12 imaging modes. It can provide users with the long time stable data support services. Fig. 1 is a scene of SAR image that is obtained by the GF-3 satellite based on the satellite parameters, image processing and relative radiometric correction. It preserves the amplitude, phase and polarization information. Table 1 is the parameters of the above image [10,11].

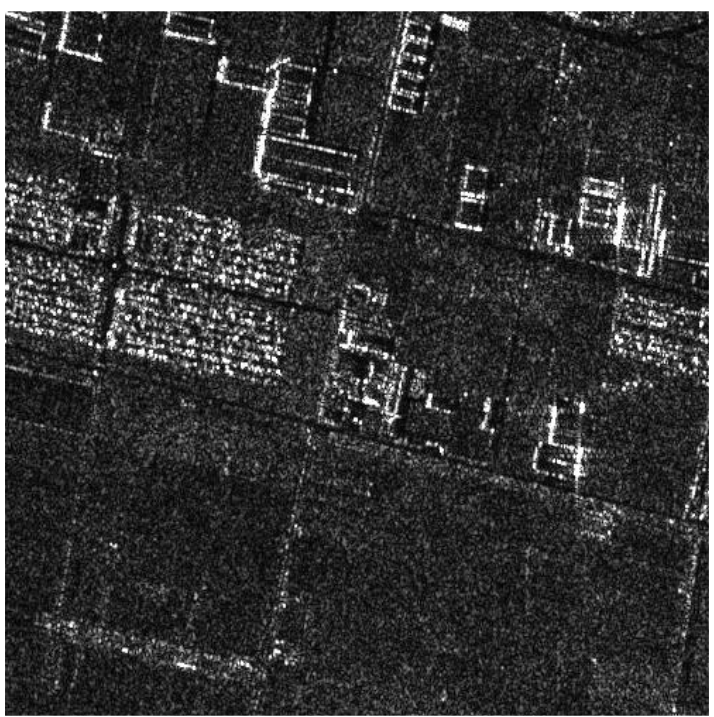

Fig 1. GF-3 SAR image

\begin{tabular}{|c|c|c|c|c|}
\hline $\begin{array}{l}\text { ground } \\
\text { receivin } \\
\text { g station. }\end{array}$ & $\begin{array}{l}\text { imaging } \\
\text { model }\end{array}$ & polarization & resolution & $\begin{array}{l}\text { imaging } \\
\text { position }\end{array}$ \\
\hline KAS & FSI & HH & $5 \mathrm{~m}$ & $\begin{array}{l}\text { E113.4 } \\
\text { N34.7 }\end{array}$ \\
\hline
\end{tabular}

Table 1. The parameters of Fig 
The data of GF-3 SAR images are variety. But the type of GF-3 SAR image data usually used are amplitude image and intensity image. If get a complex image, we need to convert it into other types. In general, we extract the real image and the imaginary image from the complex image. Real and imaginary image composite amplitude and intensity images. Amplitude image is synthesized according to the formula 1 . Intensity image is synthesized according to the formula 2 .

$$
\begin{gathered}
A=\sqrt{{X_{r e}{ }^{2}+X_{i m}{ }^{2}}^{2}} \\
I=X_{r e}{ }^{2}+X_{i m}{ }^{2}
\end{gathered}
$$

Where, $X_{r e}$ and $X_{i m}$ are real part and imaginary part, respectively.

Here is an example of the conversion of a complex image into amplitude and intensity image. Fig 2(a) show the complex image. Fig 2(b) and Fig 2(c) show the real image and the imaginary image that are extracted from the complex image. Fig 2(d) and Fig 2(e) show the amplitude image and intensity image by data conversion from the real image and the imaginary image. It fund that the types of GF-3 SAR image are variety and there have obvious feature structures. So GF-3 SAR image is widely used.

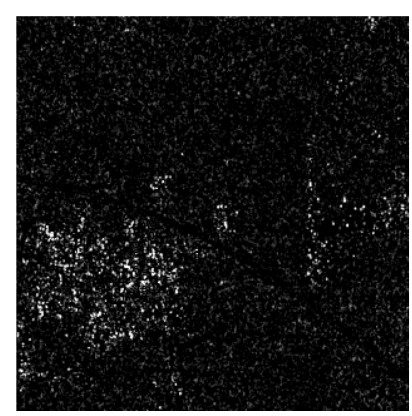

(a)

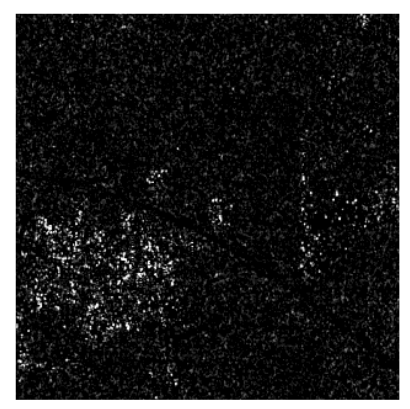

(b)

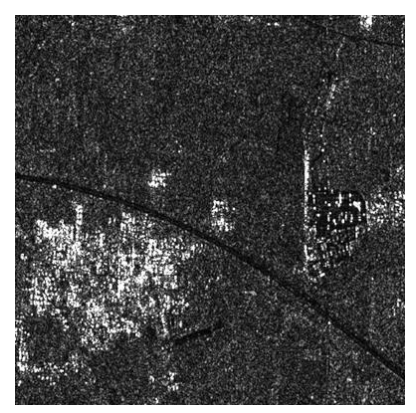

(d)

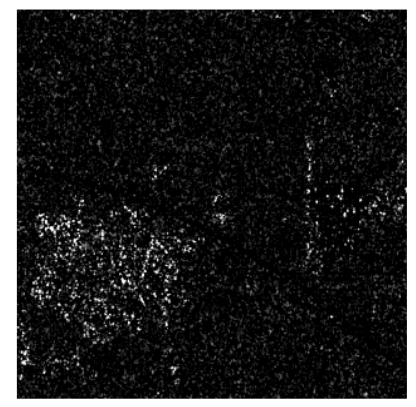

(c)

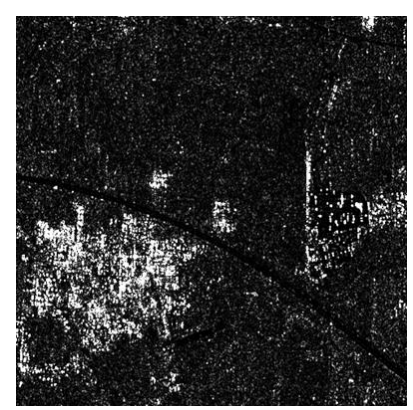

(e)
Fig 2. GF-3 SAR image: (a) Amplitude image

(b) Intensity image.

\section{SPECKLE REDUCTION ALGORITHON BASED ON LOCAL STATISTICAL CHARACTERISTICS}

If there is a large number of scatterers in the resolution cell of SAR image, all scatterers are independent and identically distributed, and there is no dominant scatterers. Then we can think that the spots are fully developed. It is generally believed that the fully developed specks are subject to the following multiplicative model [12]:

$$
Y=F \times X
$$

Where, $Y$ is the observed image, $\mathrm{X}$ is the real image, $F$ is the speckle, and it is assumed to be independent from $X$. For intensity image, $F$ obeys the Gamma distribution of unit mean. Therefore, the statistical characteristics of real image and observed image have the following relations:

$$
\mu_{X}=\mu_{Y}, C_{X}^{2}=\frac{C_{Y}^{2}-C_{F}^{2}}{1+C_{F}^{2}}
$$

Where, $\mu_{X}$ is the mean values of real images, cis the mean values of observe images, $C_{X}, C_{Y}$ and $C_{F}$ are the coefficients of variation of real images, observed images and speckle, respectively.

The most representative is the Lee filter based on the MMSE criterion. Its filtering formula is given as follows [13,14]:

$$
\begin{gathered}
\hat{X}=(1-K) \mu_{Y}+K Y \\
K=1-\frac{C_{F}^{2}}{C_{Y}^{2}}
\end{gathered}
$$

Where, $\hat{X}$ is the estimation of the real despeckled image. $K$ is a filter weighting factor.

The most representative is the Gamma MAP filter in the MAP based despeckling algorithm. The Gamma MAP filtering formula is as follows [15]:

$$
\hat{X}=\frac{(\alpha-L-1) \mu_{Y}+\sqrt{\mu_{Y}^{2}(\alpha-L-1)^{2}+4 \alpha L Y \mu_{Y}}}{2 \alpha}
$$

Where, $\alpha$ is a heterogeneous parameter that describes the degree of inhomogeneous of the real image. For the current region, it calculates the heterogeneity parameters of the real image, and use the non-linear combination of the local mean value and the gray value of the central pixel as the filter output. This is the implementation step of the Gamma MAP filter $[5,16]$. There are traditional despeckling based on local statistical characteristics of SAR images, such as Lee filter and Gamma MAP filter. However, they cannot effectively suppress the speckle in homogeneous region and edge region and cannot effectively preserve the strong edge, structure information, and the point targets. So the structural information detection based filter is constructed.

\section{ALGORITHM DESCRIPTION}

The structural information detection based filter is used. It is constructed, successively including the point target detection in the smallest window, the adaptive windowing method based on regional characteristics, and the most homogeneous sub- 
window selection. Firstly, the structural information detection algorithm classifies the local region and directly perseveres the strong edges and structures as well as the point targets. Secondly, the homogeneous region and the weak edge and structure region grow adaptively to obtain appropriate windows. Lastly, the new filtering windows are classified. For the homogeneous region, the averaged filter is directly used. For the edge and structure region, the structure information is detected and the homogeneous sub-window is selected as the final filtering region. Fig 3 shows the flowchart of our method.

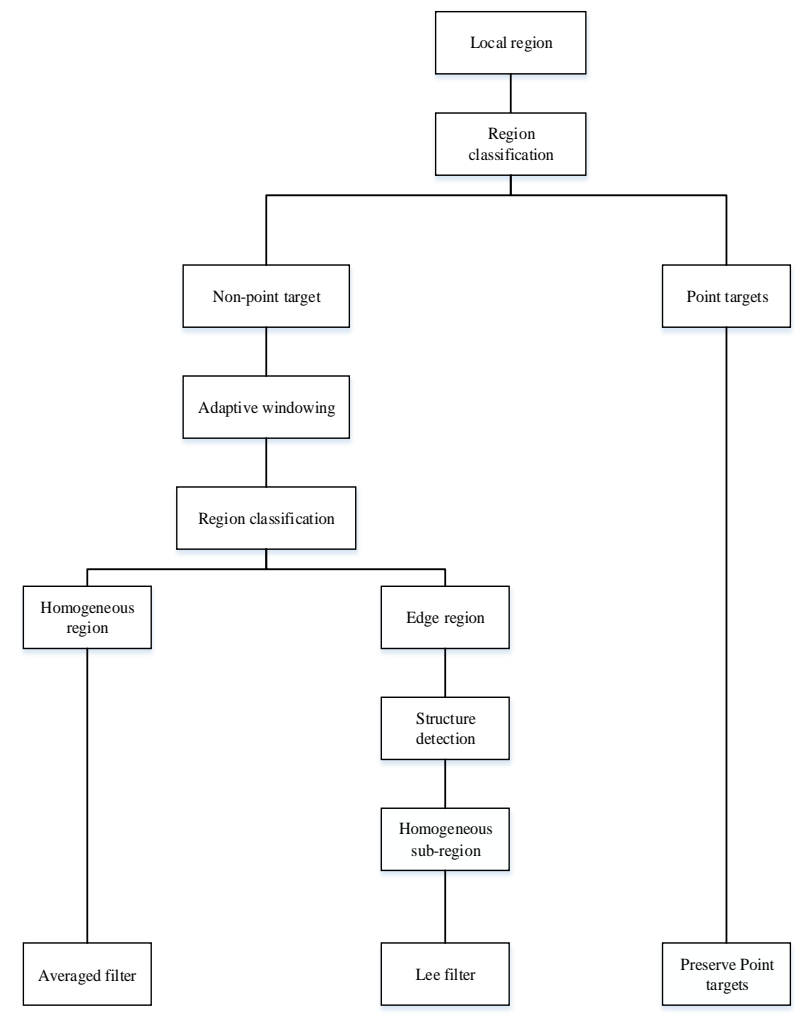

Fig 3. The flowchart of our method

\section{DESPECKLING EXPERIMENT}

There are two GF-3 SAR images to do the experiment. Fig 4 shows the GF-3 SAR image, Fig 5 and Fig 6 shows the denoised image using the classical Lee filter method, Gamma MAP filter method, and our method, respectively. It can be seen that the structural information detection based filter can smooth speckle, and preserve the structural information effectively. But the Lee filter and Gamma MAP filter cannot suppresses the speckle sufficiently.

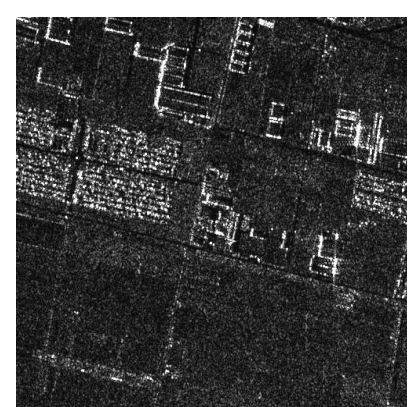

(a)

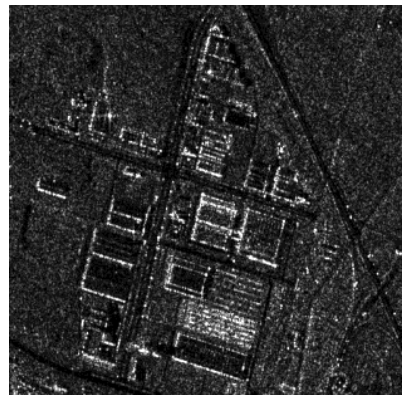

(b)
Fig 4. GF-3 SAR Image: (a) Image1, (b) Image2.

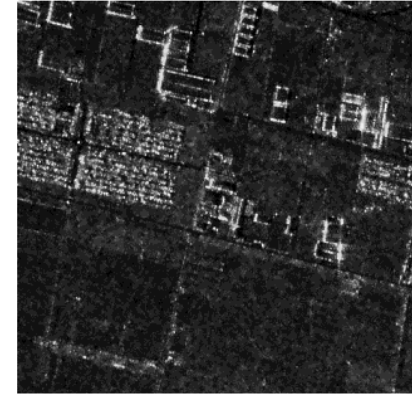

(a)

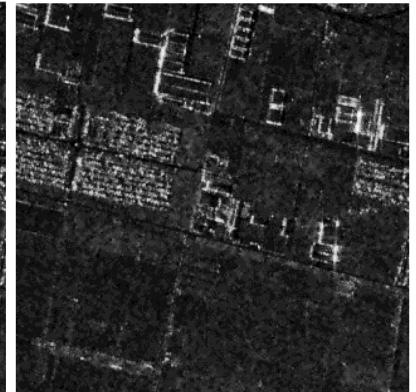

(b)

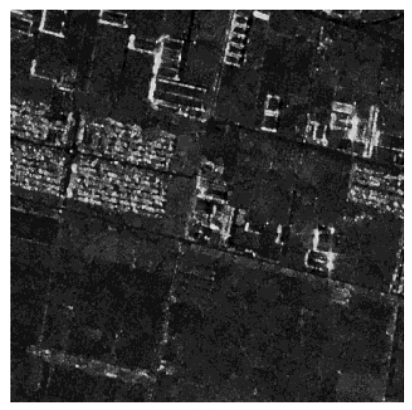

(c)

Fig 5. Despeckling result Image1: (a) Lee filter, (b) Gamma MAP filter, (c) Our method. (a)

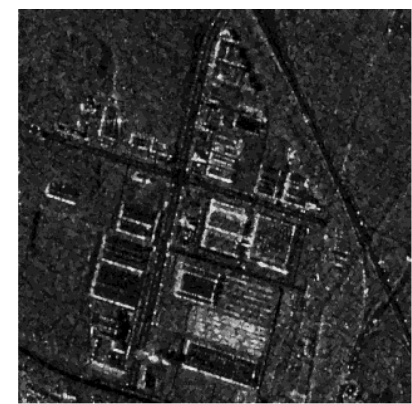

(c)

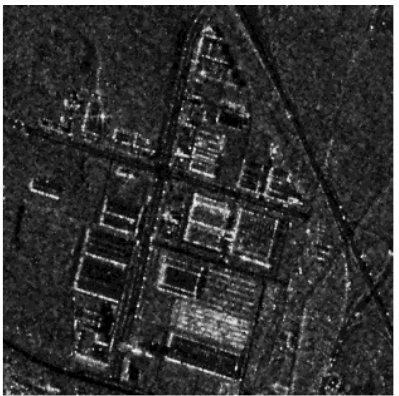

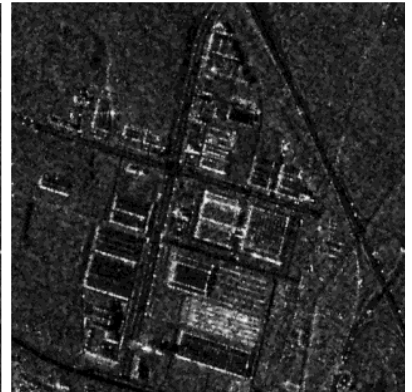

(b)
Fig 6. Despeckling result Image2: (a) Lee filter, (b) Gamma MAP filter, (c) Our method.

Some quantitative measures are provided in Table 1 to evaluate the various despeckling methods. The coefficient of variation (DCV) is an index measuring the capability of edge preserving. If the value of DCV is close to zero, it indicates that the method has better edge preserving. The DCV is given by

$$
D C V=\left|C_{\hat{X}}-C_{X}\right|
$$




$$
C_{X}=\sqrt{\frac{C_{Y}^{2}-C_{F}^{2}}{1+C_{F}^{2}}}, C_{Y}=\frac{\sigma_{Y}}{\mu_{Y}}, C_{\hat{X}}=\frac{\sigma_{\hat{X}}}{\mu_{\hat{X}}}
$$

Where, $\sigma_{Y}$ and $\sigma_{\hat{X}}$ are mean values of the observed image and the denoised image, $\mu_{Y}$ and $\mu_{\hat{X}}$ are mean values of the observed image and the denoised image, respectively.

The equivalent number of looks (ENL) is an index measuring the capability of speckle suppression. ENL is defined in (10). If the value of ENL is bigger, it means that the method leads to better speckle suppression. We can see that, compared with the traditional filters, the structural information detection based filter can well preserve the points, edges and lines as well as smooth the speckle more sufficiently[14,17].

$$
E N L=\frac{\mu_{S}^{2}}{\sigma_{S}^{2}}
$$

Where, $S$ is a uniform area of the image. $\mu_{S}$ is the mean value of pixel gray in the homogeneous region of denoised image. $\sigma_{S}^{2}$ is the standard deviation of the denoised homogeneous region.

\begin{tabular}{|c|c|c|c|}
\hline & $\begin{array}{c}\text { Gamma MAP } \\
\text { filter }\end{array}$ & Lee filter & Our method \\
\hline DCV & 0.0093 & 0.0136 & 0.0080 \\
\hline ENL & 6.67 & 5.62 & 7.91 \\
\hline
\end{tabular}

Table 2. Comparison of quantitative measures

Fig 7(a) show the speckle pattern corresponding to the three speckle filters in Fig 6, respectively [18]. The quantitative measure of speckle pattern measure the ability of suppress the speckle and edge retention of the filter. Obviously, in the Fig 7(a) and Fig 7(b), there have clearly intermittent white speckle in the edge position. However, in the Fig 7(c), this situation does not exist. So our method can effectively suppress the speckle in homogeneous region and edge region and can effectively preserve the strong edge, structure information, and the point targets compared to the Lee filter and Gamma MAP filter.

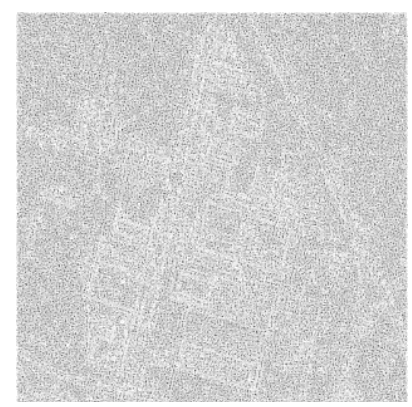

(a)

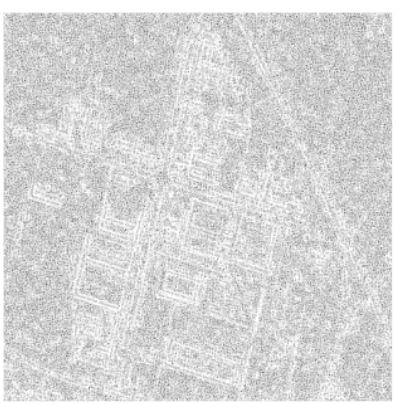

(b)

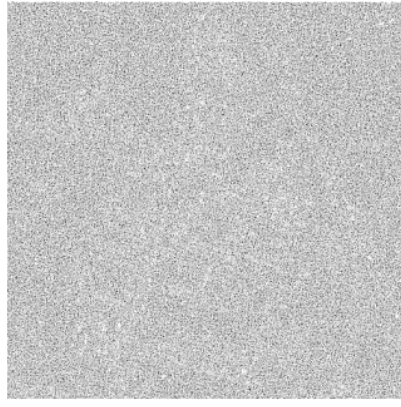

(c)

Fig 7. Speckle pattern: (a) Lee filter, (b) Gamma MAP filter, (c) Our method.

\section{CONCLUSION}

The high-resolution SAR images are rich in information and have visible feature structures such as points, edges, lines and so on. Due to the coherent imaging system, speckle appears in GF-3 SAR images, and it hinders the understanding and interpretation of images seriously. However, The traditional filters not favorable for the GF-3 SAR images. So we used structural information detection based filter. The despeckling experiments on GF-3 SAR images demonstrate that compared with the traditional filters, the used structural information detection based filter can well preserve the points, edges and lines as well as smooth the speckle more sufficiently.

\section{REFERENCES}

Oliver C, Quegan S. Understanding Synthetic Aperture Radar Images[M]. Boston, MA: Artech House, 1998.

Gomez L, Ospina R, Frery A C. Unassisted Quantitative EvaluationOf Despeckling Filters[J]. Remote Sensing, 2017: $1-24$

Janwale A P, Lomte S. Enhancement of Cotton leaves images using various Filtering techniques[C]// IEEE International Conference on Data Management, Analytics and Innovation. IEEE, 19 October 2017: $303-305$.

Lee J.S. Digital image enhancement and noise filtering by use of local statistics. IEEE Trans. Pattern Anal. Machine Intell. 1980, Mar, 2(2) : 165-168.

Baraldi A, Parmiggiani F. A refined gamma MAP SAR speckle filter with improved geometrical adaptivity[J]. Geoscience \& Remote Sensing IEEE Transactions on, 1995, 33(5) : 1245-1257.

Beijing, Beijing. The Analysis of Noise Reduction for SAR Image Based on Filters[J]. Microcomputer Information, 2012: 24-26.

Zhang Z, Sun Z. Frost Filtering Algorithm of SAR Images with Adaptive Windowing and Adaptive Tuning Factor[C]// International Conference on Advances in Mechanical Engineering and Industrial Informatics. 2015: 538-544.

Park J M, Song W J, Pearlman W A. Speckle filtering of SAR images based on adaptive windowing $[\mathrm{J}]$. IEE Proceedings - Vision, Image and Signal Processing, 2002, 146(4) : 191-197. 
The International Archives of the Photogrammetry, Remote Sensing and Spatial Information Sciences, Volume XLII-3, 2018 ISPRS TC III Mid-term Symposium "Developments, Technologies and Applications in Remote Sensing", 7-10 May, Beijing, China

Lang F, Yang J, Li D. Adaptive-Window Polarimetric SAR Image Speckle Filtering Based on a Homogeneity Measurement[J]. IEEE Transactions on Geoscience \& Remote Sensing, 2015, 53(10): 54355446.

Zhao R, Zhang G, Deng M, et al. Geometric Calibration and Accuracy Verification of the GF-3 Satellite.[J]. Sensors, 2017, 17(9): 1977.

Xiang Y, Wang F, You H. An Automatic and Novel SAR Image Registration Algorithm: A Case Study of the Chinese GF-3 Satellite[J]. Sensors, 2018, 18(2): 672 .

Liu S, Geng P, Shi M, et al. SAR Image De-noising Based on Generalized Non-local Means in Non-subsample Shearlet Domain[M]// Proceedings of the 2015 International Conference on Communications, Signal Processing, and Systems. Springer Berlin Heidelberg, 2016: 221229.

Yommy A S, Liu R, Wu A S. SAR Image Despeckling Using Refined Lee Filter[C]// International Conference on Intelligent Human-Machine Systems and Cybernetics. IEEE, 2015 : 260-265.

Liu S, Liu M, Li P, et al. SAR Image Denoising via Sparse Representation in Shearlet Domain Based on Continuous Cycle Spinning[J]. IEEE Transactions on Geoscience \& Remote Sensing, 2017(99) : 1-8

Li T, Xu Y, Zhu D, et al. Preprocessing Method for SAR Images Based on Edges-preserving[J]. Computer Measurement \& Control, 2012: 2782-2784.

Lang W, Zhang P, Wu J, et al. Incidence Angle Correction of SAR Sea Ice Data Based on Locally Linear Mapping[J]. IEEE Transactions on Geoscience \& Remote Sensing, 2016, 54(6) : 3188-3199.

Hu S, Ma X, Liu S, et al. SAR image de-noising based on non-local similar block matching in NSST domain[C]// IEEE, International Conference on Signal Processing. IEEE, 2017 : 832-836.

Hou $\mathrm{B}$, Zhang $\mathrm{X}, \mathrm{Bu} \mathrm{X}$, et al. SAR Image Despeckling Based on Nonsubsampled Shearlet Transform[J]. IEEE Journal of Selected Topics in Applied Earth Observations \& Remote Sensing, 2012, 5(3) : $809-823$ 